\title{
Biodegradable and bioactive porous polyurethanes scaffolds for bone tissue engineering
}

\author{
Mei-Na Huang ${ }^{1}$, Yuan-Liang Wang ${ }^{{ }^{*}}$, Yan-Feng Luo ${ }^{1}$ \\ National 985 Research Center of Bioinspired Material Science and Engineering, Bioengineering College,Chongqing University, Chongqing 400030, People’s \\ Republic of China, Correspondence to Yuan-Liang Wang (wyl@cqu.edu.cn). Tel. /fax: +86-23-65102509. \\ Received September $22^{\text {nd }}, 2008$; revised November $12^{\text {th }}, 2008$; accepted November $19^{\text {th }}, 2008$
}

\begin{abstract}
Biodegradable porous polyurethanes scaffold have themselves opportunities in service, including controlled degradation rate, no-toxic degradation products. However, polyurethanes are lack of bioactive groups, which limits their application. This review gives the common modification methods, surface functionalization and blending modification. In finally, the review puts forward to the bulk modification as a new method to enhance the bioactivity of polyurethanes.
\end{abstract}

Keywords: Polyurethanes, Bioactivity, Biodegradation, Bone Repair

\section{INTRODUCTION}

Currently, tissue engineering involving synthetic materials offers a practical approach for bone repair and regeneration. In this approach, a 3-D porous biodegradable scaffold is beneficial to guide cell attachment, proliferation and tissue regeneration [1,2]. Therefore, a number of researchers are interested in developing biodegradable polymeric scaffolds for bone engineering repair $[3,4,5,6]$. Polyurethane, which concludes the polyurethane urea elastomer, is regarded as a kind of bone repair materials for its nice mechanical property and their special shape memory function.

Biodegradable polyurethanes, made from degradable polyester/polyether with hydrophilic group of ether bond, aliphatic diisocyanate, having the hydrophobic group of alkly and chain extenders $[7,8]$. Due to these special group, polyurethanes have controlled degradation rate, in general, the degradation time can reach to some months with changing of the ratio polyester/polyether to diisocyanate $[7,9]$, which fits to the growth rate of osteoblast. Moreover, the degradation give rise to non-toxic products, which will not produce side effect for body. Besides polyester/polyether and diisocyanate, chain extender is also a key factor. In order to regulate the $\mathrm{pH}$ of degradation products, and avoid the acid auto-catalytic effect in the degradation process, and then further controlling the easily control of degradation rate, some researches choose diamines [10]. Guan et al [4] synthesized (poly (etherurethane urea), PEUU) with PCL and 1, 4-diisocyanatobutane (BDI) and putrescine. And then, PEUU was made into highly porous, biodegradable polyurethane scaffold for tissue engineering. In this study, BDI was used, since it could release putrescine, a polyamine that is essential for cell growth and proliferation. Zhang et al [11] synthesized polyurethane by reacting of highly pure lysine diisocyanate with glucose, which resulted in major degradation products lysine and glucose (LDIglucose), and then completely degradate and enter into human circulation system.

The degradation mechanisms of polymers are important and need to be investigated further. Non-toxic degradation products are necessary and, moreover, mechanical properties are also influenced by degradation mechanisms. LDI-glucose [11] polymer, for example, is degraded by hydrolysis of urethane bonds to liberate lysine, glucose, ethanol, and $\mathrm{CO}_{2}$. Ethanol could inhibit cell-cell adhesion, but a study reported that concentrations less than $30 \mathrm{mM}$ are harmless to the cell. Moreover, in contrast to PLA and PLGA degradation mechanisms, the study showed that the degradation of polyurethane with diamine no significant increase in $\mathrm{pH}$ of the solution. PEUU degradation products were also shown to be non-toxic to endothelial cells. The polymer showed a linear degradation with no signs of autocatalytic effects when compared to PLA or PLGA degradation behaviour. In addition, regulating ratio of polyester/polyether to diisocyanate can change the molecular weight of polyurethane, and then control their degradation rate. The two regulation methods make it be balance with growth of cell/tissue and realize the real tissue engineering repair.

However, polyurethanes as a potential, biodegradable materials are lack of bioactive groups, which limits their applications. Therefore, how to ensure biodegradation and bioactive of polyurethane are two key factors for it's application in bone repair $[12,13]$. A further requirement for scaffold, particularly used for bone engineering, is controllable interconnected porosity for cells to grow into the desired physical form and to compete vascularization of the ingrown tissue [12]. Other highly desirable 
features concerning the scaffold processing are near-net -sHAe fabrication and scalability for cost-effective industrial production $[12,14]$.

In the paper, we only discuss how to enhance the bioactivity of porous polyurethane scaffold. In general, bioactive functionalization methods of polyurethanes can be concluded to three major design strategies [15,16,17, $18,19]$. One approach is blending the polyurethanes with tricalcium phosphate/ hydroxyapatite or other inorganic ceramic $[16,17,18,19]$. Various bioactive factors further enhance the cellular compatibility. The inorganic ceramic have another advantage, the function of bone induction and the conduction $[16,17,18,19]$. The other approach involves endowing the biomaterials with bioactivity by incorporating soluble bioactive molecules, such as growth factors and plasmid DNA, into biomaterial carriers so that the bioactive molecules can be released from the materials and trigger or modulate new tissue formation [20,21,22]. The last one is incorporation of cell-binding peptides into biomaterials via chemical or physical modification. The cell-binding peptides include a native long chain of extracellular matrix (ECM) proteins as well as short peptide sequences derived from intact ECM proteins that can incur specific interactions with cell receptors [15,23,24,25] This paper reviews above methods and focuses on their opportunities as a kind of bone repair materials, and puts forward a new method to improve the bioactivity of biodegradable polyurethanes.

\section{BIOACILITY OF POLYURETHANE}

Tissue engineering applies methods from materials engineering and life sciences to artificial construction new tissue. Two common approaches are transplanting the biomaterials with cell [26] or the biomaterials with some bioactive factor/bioactive substance for the cell homing to realize restoration. Facing the complex biological and sensitive human body, requirements of biomaterials are extremely challenging. The First and most, compared to other bioactive materials, polyurethanes are lack of bioactive factors and cytocompatibility [27], which can be well solved by introduction of bioactive substances, including the inorganic phosphate, growth factors and extracellular matrix.

\subsection{Introduction of Inorganic Phosphate Into Polyurethanes}

Hydroxyapatite, glasses, glass-ceramics or calcium phosphates having similar components with natural bone $[14,20]$, are important categories of bioactive materials. Coating and blending are the most common methods to modify polymer with inorganic phosphate. Biomimetic method is a chemical modification with inorganic phosphate $[28,29,30]$.

Hydroxyapatite (HA), the most important inorganic phosphate, has been extensively investigated over the past few decades as a biomedical material. It can be designed as a bioactive material, besides it is similar com- position with natural bone, osteoconducive, osteoinductivity, biodegra-dability, high mechanical strength and their medical products such as screws, plates and rods have been commercial forms a strong bond to natural bone in vivo $[31,32,33]$. Moreover, the introduction of HA can regulate the $\mathrm{pH}$ of biomaterials. Above properties of hydroxyapatite and other inorganic phosphate can induct the growth of bone and prevent the inflammatory reaction $[31,32,33,34]$.

Rezwan, K. et al [13] reviewed the function of bioactive glasses, glass-ceramics and the calcium phosphates or HA in the enhancement of the bioactivity of polyurethanes. It has been found that reactions on bioactive glass surfaces can release critical concentrations of soluble $\mathrm{Si}, \mathrm{Ca}, \mathrm{P}$ and $\mathrm{Na}$ ions, depending on the processing route and particle size. The released ions induce intracellular and extracellular responses. One key reason that makes bioactive glassed-correlation material is the possibility of controlling a range of chemical properties and thus the rate of bioresorption. Park, Y.S. et al [35] investigated the fabrication method of a three-dimensional reticulated scaffold with interconnected pores of several hundred micrometers using calcium phosphate glass in the system of $\mathrm{CaO}-\mathrm{CaF}_{2}-\mathrm{P}_{2} \mathrm{O}_{5}-\mathrm{MgO}-\mathrm{ZnO}$ and a polyurethane sponge as a template. It is thought that this kind of biodegradable glass scaffold combined with osteogenic cells has potential to be studied further as a tissue engineered bone substitute. The structure and chemistry of glasses, in particular sol-gel derived glasses, can be tailored at a molecular level by varying either composition, or thermal or environmental processing history.

Above inorganic phosphate is important bioactive modification material, however, current technology is difficult to solve the compatibility between inorganic phosphate and polyurethanes. It is difficult to make a uniform matrix, particularly, the current coating/blending methods, which result in that it is difficult to form a uniform matrix, particularly, the content of inorganic ceramic is high [36,37]. Some researches found that some of HA/PLA composites lost their strengths rapidly in physiological environment and failures occur mainly at the interface of HA and the polymer matrix. Two main reasons may take responsibility for these interfacial failures: one is lack of effective adhesion between ceramic phase and polymer matrix; the other is self-catalytic degradation of hydroxyl groups on HA surfaces to polymer main chains. The structure of polyurethanes/HA is similar to HA/PLA, which may result in the same interface separation. For solving the problem, Xian, Y.M adopted chemical reaction to produce HA crystal on the polymer surface, the chemical reaction to make inorganic phosphate in the surface polymer can solve the interface separation, however, another problem appeared [30]. The reaction of making $\mathrm{HA}$ /polymers crystal is similar to the biomimetic calcification, which lasted for more than one week, and then make negative effect on the polymers. Moreover, the products can not ensure the crystal structure. 
Introducing inorganic phosphate can not wholly solve the bioactivity problem. Some researchers use bioactive factor, which can react with polymers to enhance their bioactivity $[38,39,40]$, such as RGD, moreover, the bioactive factors is important for cell homing.

\subsection{Surface Modification of Porous Polyure- thanes Scaffold with Bioactive Factors}

\subsubsection{Arg-Gly-Asp(RGD) Modified Biomimetic Polyurethanes}

In an effort to improve the adhesion and retention of cells to polymer scaffolds, researches typically coated with various extracellular matrix proteins [40,41,42]. These studies highlight that extracellular proteins played an important role in attachment and spreading of cells to surface, where specific domains on cell membrane bind directly with extracellular matrix moleculaes via integrins $[43,44]$. A number of specific cell-recognition sequences have been identified, the most extensively studied sequence being the arginine-glycine-aspartic acid (RGD) motif present in matrix molecules such as vitronectin, fibronectin, laminin and collagen, fibrillin $[40,45,46,47]$.

RGD peptide is one of the major bioactive factor to design biomimetic polyurethanes and has been widely researched in recent years $[38,39,40]$. In order to provide a stable linking, RGD peptides should be covalently attached to polymer via functional groups like hydroxyl-, amino-, or carboxyl-groups. Some polyurethanes are amino-terminated $[4,38]$, which can react with the carboxyl-groups of RGD, with 1,3-Dicyclohexylcarbodiimide (DCC) as catalyst. Other polyurethanes are hydroxyl-terminated, the hydroxyl-also can react with the carboxyl-group of RGD [48].

Moreover, in order to enhance the surface functionalization, polymeric materials, such as polyurethane must be functionalized before bioactive peptides or proteins are immobilized on their surfaces [44]. In general, the functionalization can be realized by a variety of means, either by introduced the multi-functional groups monomer or polymer[39], or by subsequent surface modification by plasma treatment [45] ozone oxidation [46] surface graft polymerization [40] or site-specific reactions [47]. Here, we put emphasis on two examples to demonstrate the successful application of linking group in surface modification. One example [39], the difunctional spacer molecule-diisocyanate is introduced as the linking group of polyurethane film and RGD, realizing the surface functionalization of polyurethane. Another example, Jozwiak, A.B [40] used two steps to enhance the introduction rate. First, the multi-amino group-polyethyleneimine (PEI) is introduced, a medium sized molecular weight branched form of PEI was used here in order to provide a large number of reactive primary amine groups and enhance its entrapment within the polyurethane surface. Second, introducing the dextran, which is functional spacer molecule and can link the RGD easily.

\subsubsection{Growth Factors Modified Biomimetic Poly- urethanes}

Chemotaxis, proliferation, differentiation and matrix synthesis are essential in natural tissue/organ development and wound healing [45]. Owing to the rapid advances in recombinant technology and the availability of large scale manufacturing of cytokines and growth factors, many recent tissue engineering strategies have turned to specific growth factors to stimulate cellular activity in vitro and to improve functional neotissue formation in vivo $[47,48]$. Characteristic of these bioactive factors is that they can effective release at specific site and realize the function of improving cell proliferation and recruitment $[46,49]$. Incorporation of angiogenic growth factors such as basic fibroblast growth factor (bFGF) and vascular endothelial growth factor (VEGF), among others, into scaffolds for controlled release has been shown to promote lacal angiogenesis [50]. Platelet-derived growth factor (PDGF) has been demonstrated to stimulate proliferation and recruitment of both periodontal ligament and bone cells in vitro. In vivo study also showed that PDGF-BB enhances the ability of healing [46].

There are many methods to incorporate growth factors into synthetic scaffolds, such as absorbing growth factor to scaffold, and blending growth factor containing microspheres into the scaffold [46], or directly mixing growth factor containing protein powder into the scaffold during processing [50]. However, absorbing growth factors onto the scaffold has the drawback of low loading efficiency and rapid releasing, which may be associated with in bioactivity due to harsh solvents such as hexane [46] or methylene chloride [51]. Incorporating growth factor directly into the scaffold can potentially avoid these shortcomings.

Whether or not has bioactivity of the released bioactive factors is an essential problem. Bioactivity of the factors can be assessed in two methods [47,50,52]. First, bioactivity of the released factor can be determined through the direct method-human gingival fibroblase DNA synthesis as measured by specific composition [46] Second, the bioactivity is assessed in terms of its ability to stimulate the growth of cells [50,52].

\section{CONCLUTION AND PRESPECT}

\subsection{Possibility and Challenge of Bulk Modi- fication for Polyurethanes}

Besides above methods, how can we improve bioactivity of polymers? Now, a great wealth of knowledge about the biology of integrin mediated cell adhesion has proved that the modification of polyurethanes with RGD peptides or other bioactive factors are useful tool to design bioactive porous scaffolds that can provide biological cues elicit specific cellular responses and direct new tissue formation. However, the surface modification has some limitations. Since surface modification has been performed on well-defined model surfaces and the 
evaluation of cell behavior on material has been conducted under serum free media, the results may not properly indicate complicated events associated with in vivo environments. Even though some model surfaces may be useful to provide fundamental knowledge to understand cell behavior through specific binding, they may not be directly used as tissue engineering scaffolds.

If we use bulk designing of polyurethanes, incorporated RGD or collagen may result in recognition sites is present not only on the surfaces but also in the bulk of the materials. Niu, X.F. et al [53] review the bulk modification, which describe the bulk modification of biomaterials is beneficial to tissue engineering applications where injectable biomimetic materialsare required to match the complex HA of native tissue at defect sites. Cook et al [54] and Barrera et al [55] conducted a lot of investigations in understanding the effects of bulk modification via RGD peptides. They synthesized RGD bulk modified poly (lactic acid-co-lysine) and successfully blended it with PLA to fabricate a thin film. When this film was exposed to endothelial cell suspended media for $4 \mathrm{~h}$, the specific function of RGD was maintained to facilitate cell spreading.

Polyurethanes are the biomaterials with hydroxylterminated and amine-terminated. The RGD or other peptide can react with the terminal group of polyurethanes, which may result in bioactive polyurethanes. My laboratory chose the bulk modification to introduce the bioactive factor, such as RGD/MGF, and then emulsion/freeze drying mean was adopted to make porous polyurethane scaffold with bioactivity polyurethane.

\subsection{The Possibility of Introduction of Inor- ganic Phosphate by Chemical Reaction}

Inorganic phosphate is important component of natural bone, however, current technology is difficult to solve the compatibility between the inorganic phosphate and polyurethanes.

How to introduce the inorganic phosphate, and at the same time avoid above disadvantage is a key problem for enhancing the stability of polyurethane/inorganic phosphate composition. In order to overcome these limitations of composite, covalently attached the inorganic phosphate to polyurethanes by linking group may be a feasible method. Linking group should easily react with the hydroxyl-from inorganic phosphate and the carboxylor amino-group from polyurethanes. Silane derivatives are used as modification molecular to link hydroxyl groups (-OH) in HA surface to polymer main chain, which is carried out via direct reactions of -OR groups on HA surfaces. At the same time, other functional groups $\left(-\mathrm{NH}_{2}\right)$ of silane derivatives may further react towards the terminal groups carboxylic group or hydroxyl group. Moreover, glutaraldehyde [43] may be the important cross-linking agent. In addition, in order to ensure the homogeneity of composite, the effective connection of emulsion blending-chemical crosslinking may be an efficient method [44].
For realizing biodegradation, bioactivity and mechanical property of the bone repair materials, the paper puts forward two methods to make the biodegradable materials, which are equipped with the uniform structure and bioactive components.

\section{ACKNOWLEDGEMENT}

The authors gratefully acknowledge the financial support from the "Eleven-Five" National Science and Technology Support Program of China (2006BA103B04).

\section{REFERENCES}

[1] J. R. Hench, (2003) Regeneration of trabecular bone using porous ceramics. Current Opinion in Solid State and Materials Science, 7, 301-307.

[2] Z. H. Zhou, J. M. Ruan, (2008) Preparation and bioactivity of sol-gel macroporous bioactive glass. Journal of University of Science and Technology, 15, 290-298.

[3] J. J. Guan, W. R. Wagner, (2005) Synthesis, characterization and cytocompatibility of polyurethane urea elastomers with designed elastase sensitivity. Biomacromolecules, 6, 2833-2842.

[4] J. J. Guan, K. L. Fujimoto, (2005) Preparation and characterization of highly porous, biodegradable polyurethane scaffolds for soft tissue applications. Biomaterials, 26, 3961-3971.

[5] Y. Wang, G. B. Ameer, (2002) A tough biodegradable elastomer. Nature Biotechnology, 20, 602-606.

[6] K. E. Healy, A. Rezania, (1999) Designing biomaterials to direct biological responses. Annals of the New York Academy of Sciences, 875, 24-25.

[7] S. A. Guelcher, K. Gallagher, (2005) Synthesis of biocompatible segmented polyurethanes from aliphatic diisocyanates and diurea diol chain extenders. Acta Biomaterialia, 1, 471-484.

[8] K. D. Kavlock, T. W. Pechar, (2007) Synthesis and characterization of segmented poly(esterurethane urea) elastomers for bone tissue engineering. Acta Biomaterialia, 3, 475-484.

[9] S. A. Guelcher, (2008) Synthesis, mechanical properties, biocompatibility, and biodegradation of polyurethane networks from lysine polyisocyanates. Biomaterials, 29(12), 1762-1775.

[10] Y. F. Luo, Y. L. Wang, (2008) Evaluation of the cytocompatibility of butanediamine and RGDS-grafted poly (d, 1-lactic acid). European Polymer Journal, 44, 1390-1402.

[11] J. Y. Zhang, E. J. Beckman, (2002) Synthesis, biodegradability, and biocompatibility of lysine diisocyanate-glucose polymers. Tissue Engineering, 8(5), 771-785.

[12] X. Miao, Y. Hu, (2004) Porous calcium phosphate ceramics prepared by coating polyurethane foams with calcium phosphate cements. Materials Letters, 58, 397-402.

[13] K. Rezwan, Q. Z. Chen, (2006) Biodegradable and bioactive porous polymer/inorganic composite scaffolds for bone tissue engineering. Biomaterials, 27(18), 3413-3431.

[14] M. Gelinsky, P. B. Welzel, (2008) Porous three-dimensional scaffolds made of mineralised collagen: Preparation and properties of a biomimetic nanocomposite material for tissue engineering of bone. Chemical Engineering Journal, 137, 84-96.

[15] H. Shin, S. Jo, (2003) Biomimetic materials for tissue engineering. Biomaterials, 24, 4353-4364.

[16] M. Bil, J. Ryszkowska, (2007) Bioactivity of polyurethane-based scaffolds coated with Bioglass. Biomedical Materials, 2(2), 93-101.

[17] A. Chetty, T. Steynberg, (2008) Hydroxyapatite-coated polyurethane for auricular cartilage replacement: an in vitro study. Journal of Biomedical Materials Research A, 84 (2), 475-482.

[18] X. Huang, X. Miao, (2007) Novel Porous Hydroxyapatite Prepared by Combining $\mathrm{H}_{2} \mathrm{O}_{2}$ Foaming with PU Sponge and Modified with PLGA and Bioactive Glass. Journal Biomaterials Applications, 21(4), 351-374. 
[19] C. Vitale-Brovarone, E. Verne, (2007) Development of glassceramic scaffolds for bone tissue engineering: Characterisation, proliferation of human osteoblasts and nodule formation. Acta Biomaterialia, 3, 199-208.

[20] M. J. Whitaker, R. A. Quirk, (2001) Growth factor release from tissue engineering scaffolds. Journal of Pharmacy and Pharmacology, 53, 1427-1437.

[21] T. P. Richardson, W. L. Murphy, (2001) Polymeric delivery of proteins and plasmid DNA for tissue engineering and gene therapy. Gene Expression, 11, 47-58.

[22] J. E. Babensee, L. V. McIntire, (2000) Growth factor delivery for tissue engineering. Pharmaceutical Research, 17, 497-504.

[23] H. Shin, S. Jo, (2002) Modulation of marrow stromal osteoblast adhesion on biomimetic oligo[poly-thylene glycol) fumarate] hydrogels modified with Arg-Gly-Asp peptides and a poly (ethyleneglycol) spacer. Journal of Biomedical Materials Research, 61, 169-179.

[24] Y. Suzuki, M. Tanihara, (2000) Alginate hydrogel linked with synthetic oligopeptide derived from BMP-2 allows ectopic osteoinduction in vivo. Journal of Biomedical Materials Research, 50, 405-409

[25] F. Buket Basmanav, G. T. Kose, (2008) Sequential growth factor delivery from complexed microspheres for bone tissue engineering. Biomaterials, 29, 4195-4204.

[26] C. M. Hill, Y. H. An, (2007) Osteogenesis of Osteoblast Seeded Polyurethane-Hydroxyapatite Scaffolds in Nude Mice. Macromolecular Symposium, 253, 94-97.

[27] G. Ryan, A. Pandit, (2006) Fabrication methods of porous metals for use in orthopaedic applications. Biomaterials, 27, 2651-2670.

[28] T. Sakura, C. Tanaka, M. Yang, (2004) Production and characterization of a silk-like hybrid protein, based on the polyalanine region of Samia cynthia ricini silk fibroin and a cell adhesive region derived from fibronectin. Biomaterials, 25(4), 617-624.

[29] A. Rainer, S. Maria, (2008) Fabrication of bioactive glass-ceramic foams mimicking human bone portions for regenerative medicine" Acta Biomaterialia, 4, 362-369.

[30] Y. M. Xiao, D. X. Li, (2007) Preparation of nano-HA/PLA composite by modified-PLA for controlling the growth of HA crystals. Materials Letters, 61, 59-62.

[31] L. L. Hench, (1997) Sol-gel materials for bioceramic applications. Current Opinion in Solid State and Materials Science, 2, 604-610.

[32] Z. K. Hong, P. B. Zhang, (2005) Nano-composite of poly (L-lactide) and surface grafted ydroxyapatite:Mechanical properties and biocompatibility. Biomaterials, 26, 6296-6304.

[33] Qiu, X. Y., Hong, Z. K. (2005) Hydroxyapatite Surface Modified by L-Lactic Acid and Its Subsequent Grafting Polymerization of L-Lactide. Biomacromolecules, 6, 1193-1199.

[34] P. L. Lin, H. W. Fang, (2007) Effects of hydroxyapatite dosage on mechanical and biological behaviors of polylactic acid composite materials. Materials Letters, 61, 3009-3013.

[35] H. J. Moon, K. N. Kim, (2006) Effect of calcium phosphate glass on bone formation in calvarial defects of Sprague-Dawley rats. Journal of Materials Science Materials in Medicine, 17 (9), 807-813.

[36] J. Russias, E. Saiz, (2006) Fabrication and mechanical properties of PLA/HA composites: A study of in vitro degradation. Materials Science and Engineering C, 26, 1289-1295.

[37] S. M. Zhang, J. Liu, (2005) Interfacial fabrication and property of hydroxyapatite/polylactide resorbable bone fixation composites. Current Applied Physics, 5, 516-518

[38] H. J. Salacinski, G. Hamilton, (2003) Surface functionalization and grafting of heparin and/or RGD by an aqueous-based process to a poly(carbonate-urea)urethane cardiovascular graft for cellular engineering applications. Journal of Biomedical Materials Research Part A, 3(66A), 688-697.

[39] J. J. Guan, S. Michael, (2004) Biodegradable poly (ether ester urethane) urea elastomers based on poly (ether ester) triblock copolymers and putrescine: synthesis, characterization and cytocompatibility. Biomaterials, 25, 85-96.

[40] A. B. Jozwiak, C. M. Kielty, (2008) Surface functionalization of polyurethane for the immobilization of bioactive moieties on tissue scaffolds. Journal of Materials Chemistry, 18, 2240-2248.

[41] C. M. Li, C. Vepari, (2006) Electrospun silk-BMP-2 scaffolds for bone tissue engineering. Biomaterials, 27, 3115-3124.

[42] M. Nsksmura, M. Mie, (2008) Construction of multi-functional extracellular matrix proteins that promote tube formation of endothelial cells. Biomaterials, 29, 2977-2986.

[43] Y. M. Yue, K. Xu, (2008) Preparation and Characterization of Interpenetration Polymer Network Films Based on Poly (vinyl alcohol) and Poly (acrylic acid) for Drug Delivery. Journal of Applied Polymer Science, 6(108), 3836-3842.

[44] X. L. Xu, X. S. Chen, (2007) Electrospun poly (L-lactide)grafted hydroxyapatite/poly (L-lactide) nanocomposite fibers. European Polymer Journal, 43, 3187-3196.

[45] G. B. Wei, Q. M. Jin, (2006) Nano-fibrous scaffold for controlled delivery of recombinant human PDGF-BB. Journal Controlled Release, 112, 103-110.

[46] J. Y. Lee, S. H. Nam, (2002) Enhanced bone formation by controlled growth factor delivery from chitosan-based biomaterials. Journal Controlled Release, 78 (1-3), 187-197.

[47] J. A. Jansen, J. W. Vehof, (2005) Growth factor-loaded scaffolds for bone engineering. Journal Controlled Release, 101 (1-3), $127-136$.

[48] S. A. Guelcher, A. Srinivasan, (2008) Synthesis, mechanical properties, biocompatibility, and biodegradation of polyurethane networks from lysine polyisocyanates. Biomaterials, 25, 1762- 1775

[49] J. J. Guan, J. J. Stankus, (2007) Biodegradable elastomeric scaffolds with basic fibroblast growth factor release. Journal Controlled Release, 120, 70-78.

[50] J. Ziegler, U. Mayr-Wohlfart, (2002) Adsorption and release properties of growth factors from biodegradable implants. Journal of Biomedial Materials Research, 59, 422-428.

[51] T. W. King, C. W. Patrick, (2000) Development and in vitro characterization of vascular endothelial growth factor (VEGF)loaded poly (DL-lactic-co-glycolic acid)/poly(ethylene glycol) microspheres using a solid encapsulation/single emulsion/ solvent extraction technique. Journal of Biomedial Materials Research, 51, 383-390.

[52] F. Bono, P. Rigon, (1997) Heparin inhibits the binding of basic fibroblast growth factor to cultured human aortic smooth-muscle cells. Biochemical Journal, 326, 661-668.

[53] X. F. Niu, Y. L. Wang, (2005) Arg-Gly-Asp (RGD) modified biomimetic polymeric materials. Journal of Materials Science and Technology, 21(4), 571-576.

[54] A. D. Cook, J. S. Hrkach, (1997) Characterization and development of RGD-peptide-modified poly (lactic acid-co-lysine) as an interactive, resorbable biomaterial. Journal of Biomedial Materials Research, 4, 513-523.

[55] D. A. Barrera, E. Zylstra, (1993) Synthesis and RGD Peptide Modification of a New Biodegradable Copolymer: Poly (lactic acid-celysine). Journal of the American Chemical Society, 115, 11010-11011 